\title{
The Origem Loop
}

\author{
X. Y. Gao and J. L. Han
}

\begin{abstract}
National Astronomical Observatories, Chinese Academy of Sciences, Jia-20 Datun Road, Chaoyang District, 100012 Beijing, PR China

e-mail: bearwards@gmail.com
\end{abstract}

Received 13 June 2012 / Accepted 6 December 2012

\begin{abstract}
Context. The Origem Loop in the Galactic anticentre was discovered in 1970s. It has been suggested that it is a large supernova remnant. One later argument is that it is a chance superposition of unrelated radio sources.

Aims. We attempt to understand the properties of the Origem Loop.

Methods. Available multi-frequency radio data were used to determine the radio spectra of different parts of the Origem Loop and the polarization properties of the loop.

Results. Newly available sensitive observations show that the Origem Loop is a loop of more than $6^{\circ}$ in diameter. It consists of a large non-thermal arc in the north, which we call the Origem Arc, and several known thermal H II regions in the south. Polarized radio emission associated with the arc was detected at $\lambda 6 \mathrm{~cm}$, revealing tangential magnetic fields. The arc has a brightness-temperature spectral index of $\beta=-2.70$, indicating its non-thermal nature as a supernova remnant. We estimate the distance to the Origem Arc to be about $1.7 \mathrm{kpc}$, similar to those of some $\mathrm{H}$ II regions in the southern part of the loop.

Conclusions. The Origem Loop is a visible loop in the sky, which consists of a supernova remnant arc in the north and $\mathrm{H}$ II regions in the south.
\end{abstract}

Key words. ISM: supernova remnants - radio continuum: ISM - ISM: individual objects: Origem Loop

\section{Introduction}

Several giant loops were recognized in the early radio sky maps, i.e. Loop I (Hanbury Brown et al. 1960), Loop II (Large et al. 1962), Loop III (Quigley \& Haslam 1965), and Loop IV (Large et al. 1966). By comparing the radio continuum, $\mathrm{H} \alpha$, interstellar polarization and the H I observations of the four loops, Haslam et al. (1971) did a general review of these giant structures. Berkhuijsen et al. (1971) summarized the geometric parameters of the four loops, and proposed that they were produced by supernova explosions. Besides these four giant loops, there are also loops with smaller sizes, i.e. the Lupus Loop (Gardner \& Milne 1965) and the Cygnus Loop (Walsh \& Brown 1955), which have been definitely identified as supernova remnants (SNRs) and are collected in the well-known SNR catalogue compiled by Dave Green (Green 2009).

The Origem Loop is another known Galactic radio loop discovered in 1970s by Berkhuijsen (1974) on the $178 \mathrm{MHz}$ radio map (Caswell \& Crowther 1969) in the Galactic anticentre region between the constellations Orion and Gemini. However, its nature is under debate. It was first proposed that the loop is an old SNR at a distance of about $1 \mathrm{kpc}$ with a diameter of about $5^{\circ}$ (Berkhuijsen 1974), but it was later argued by Caswell (1985) that it is a possible projection effect of several unrelated H II regions, many extra-Galactic sources and a discrete small SNR G192.8-1.1 (PKS 0607+17) with a diameter of about 80'. However, Gao et al. (2011a) have disproved that G192.8-1.1 being an SNR. They find that it is a thermal emitter by using the Urumqi $\lambda 6 \mathrm{~cm}$ (Gao et al. 2010), the Effelsberg $\lambda 11 \mathrm{~cm}$ (Fürst et al. 1990), and the Effelsberg $\lambda 21 \mathrm{~cm}$ (Reich et al. 1997) survey data. Probably because of its large size, there were few followup observations of the Origem Loop after Berkhuijsen (1974).
Caswell (1985) discussed the region of G192.8-1.1 and some nearby H II regions, but did not study the northern part of the Origem Loop. Krymkin \& Sidorchuk (1988) made brightness temperature scans of nearly the entire loop with the UTR-2 and RATAN 600 radio telescopes at five frequencies, from $14.7 \mathrm{MHz}$ to $3950 \mathrm{MHz}$. They claimed to have discovered a new feature, namely GR $0625+16$, as another possible discrete SNR besides the "SNR" G192.8-1.1. However, the GR 0625+16 corresponds exactly to the northern arc of the Origem Loop.

High quality multi-frequency radio survey data with sufficient sensitivity and angular resolution are now available, which can be used to investigate the properties of the Origem Loop. We introduce in Sect. 2 the data sets we use, and present the analysis in Sect. 3. A summary is given in Sect. 4.

\section{Data}

The Origem Loop clearly shows up in the $\lambda 6 \mathrm{~cm}$ total intensity and polarization images from the Sino-German $\lambda 6 \mathrm{~cm}$ polarization survey of the Galactic plane ${ }^{1}$ (Gao et al. 2010), which motivated us to seek a better understanding of this large structure. Other public radio data are available from the Effelsberg $\lambda 11 \mathrm{~cm}$ (2.7 GHz, Fürst et al. 1990) and $\lambda 21 \mathrm{~cm}(1.4 \mathrm{GHz})$ Galactic plane survey (Reich et al. 1997), which can be downloaded from the survey sampler of the Max-Planck-Institut für Radioastronomie (MPIfR) ${ }^{2}$, the WMAP 7-year $K$-band $(22.8 \mathrm{GHz}, \lambda 1.3 \mathrm{~cm})$ survey data (Jarosik et al. 2011) retrieved

\footnotetext{
1 http://zmtt.bao.ac.cn/6cm/

2 http://www.mpifr.de/old_mpifr/survey.html
} 
Table 1. Parameters of the survey data for the images of the Origem Loop.

\begin{tabular}{lcccc}
\hline \hline Surveys & $\begin{array}{c}\text { Frequency } \\
(\mathrm{GHz})\end{array}$ & $\begin{array}{c}H P B W \\
\left({ }^{\prime}\right)\end{array}$ & $\begin{array}{c}\mathrm{rms} \\
\left(\mathrm{mK} T_{\mathrm{b}}\right)\end{array}$ & References \\
\hline Urumqi $\lambda 6 \mathrm{~cm}$ & 4.8 & 9.5 & $1.2(\mathrm{TP}) / 0.5(\mathrm{PI})$ & Gao et al. (2010) \\
Effelsberg $\lambda 11 \mathrm{~cm}$ & 2.7 & 4.3 & $18.0(\mathrm{TP})$ & Fürst et al. (1990) \\
Effelsberg $\lambda 21 \mathrm{~cm}$ & 1.4 & 9.4 & $22.0(\mathrm{TP})$ & Reich et al. (1997) \\
WMAP $\lambda 1.3 \mathrm{~cm}$ & 22.8 & 52.8 & $0.07(\mathrm{TP}) / 0.06(\mathrm{PI})$ & Jarosik et al. (2011) \\
DRAO $\lambda 21 \mathrm{~cm}$ & 1.4 & 36.0 & $12.0(\mathrm{PI})$ & Wolleben et al. (2006) \\
EMLS $\lambda 21 \mathrm{~cm}$ & 1.4 & 9.35 & $15.0(\mathrm{TP})$ & Uyaniker et al. (1998) \\
\hline
\end{tabular}

Notes. TP: total power, PI: polarization intensity.

from the website of $\mathrm{NASA}^{3}$, the $\lambda 21 \mathrm{~cm}$ Effelsberg Medium Latitude Survey (EMLS) data (Uyanıker et al. 1998, 1999), and the DRAO $\lambda 21 \mathrm{~cm}$ polarization survey data (Wolleben et al. 2006), both of which were also obtained from the survey sampler of MPIfR. In the following discussions, we use the observing wavelength to indicate the data set. Specifically the $\lambda 21 \mathrm{~cm}$ data stands for the Effelsberg Galactic plane survey data (Reich et al. 1997), unless special statements are made. The angular resolution is 9.5 for the $\lambda 6 \mathrm{~cm}$ image, 4.3 for $\lambda 11 \mathrm{~cm}$ image, 9.4 for $\lambda 21 \mathrm{~cm}$ image, $52 ! .8$ for $\lambda 1.3 \mathrm{~cm}, 9.35$ for EMLS $\lambda 21 \mathrm{~cm}$, and $36^{\prime}$ for DRAO $\lambda 21 \mathrm{~cm}$ images. Among them, the $\lambda 6 \mathrm{~cm}$ and $\lambda 1.3 \mathrm{~cm}$ observations provided both total intensity and polarization measurements, the DRAO $\lambda 21 \mathrm{~cm}$ data provided only the polarization image, while the rest give only the total intensity maps. The Effelsberg $\lambda 21 \mathrm{~cm}$ Galactic plane survey (Reich et al. 1997) has a latitude limit of $b= \pm 4^{\circ}$. Therefore, we used the EMLS data to fill the blank region above $b=4^{\circ}$, but we do not have any data for the region below $b=-4^{\circ}$. Basic parameters of these data sets are summarized in Table 1. As done by Gao et al. (2011b), the "background filtering" technique developed by Sofue \& Reich (1979) was applied to $\lambda 6 \mathrm{~cm}, \lambda 11 \mathrm{~cm}$, and $\lambda 21 \mathrm{~cm}$ images to separate the unrelated large-scale Galactic diffuse emission from the Origem Loop emission. A twisted hyper plane defined by the corner mean values of each image was subtracted to find the local zero level around the Origem Loop. The final $\lambda 6 \mathrm{~cm}, \lambda 11 \mathrm{~cm}, \lambda 21 \mathrm{~cm}$ total intensity images of the Origem Loop are shown in Fig. 1. The $\lambda 11 \mathrm{~cm}$ image was convolved to an angular resolution of 9.5 to get a higher signal-to-noise ratio.

\section{Results}

Radio images in Fig. 1 at three different wavelengths resemble each other in structures. At $\lambda 6 \mathrm{~cm}$, the circle that indicates the loop in Fig 1a has a radius of $200^{\prime}$ and is centred at $\ell=194^{\circ} .7, b=-0.2$. These values are different from those of Berkhuijsen (1974), because we included the region below $\delta=14^{\circ}$ (B1950), which was not included in the $178 \mathrm{MHz}$ map used by Berkhuijsen (1974). Our new sensitive measurements enable us to detect fainter and more extended emission near the boundary of the Origem Loop than ever before. The loop consists of four major parts: an elongated arc structure extending from $\ell=197^{\circ} .6$ to $\ell=192^{\circ} .1$ in the north, which can also be recognized in the $178 \mathrm{MHz}$ map shown by Berkhuijsen (1974); the $\mathrm{H}$ II region BFS 52; and two complexes formed by several known H II regions, i.e. SH 2-261, SH 2-254 to SH 2-258, the object G192.8-1.1 in the south and southwest; and another group of H II regions SH 2-268, SH 2-270 in the southeast. We marked the names of these known H II regions in Fig. 2.

\footnotetext{
3 http://lambda.gsfc.nasa.gov/product/map/dr4/ maps_band_r9_iqus_7yr_get.cfm
}

\subsection{Spectral indices and their distribution}

Spectral indices and their distribution are important properties for understanding the nature of the extended radio sources. Shell-type SNRs usually have a brightness temperature spectral index of $\beta \sim-2.5\left(T_{\mathrm{b}} \sim \gamma^{\beta}\right)$, while the spectrum of an optically thin $\mathrm{H}$ II region is much flatter, normally $\beta \sim-2.1$. We derived the brightness temperature spectral index distribution of the Origem Loop (see Fig. 1d) using the $\lambda 6 \mathrm{~cm}, \lambda 11 \mathrm{~cm}$, and the $\lambda 21 \mathrm{~cm}$ images at an angular resolution of 9'.5. A systematic error of such a spectral index map comes from the uncertainties of the baselevel determination due to the foreground/background subtraction. In Fig. 1d, the spectral index map shows reasonable thermal spectra for all known H II regions, which demonstrates that the spectral map is reliable. The northern arc of the Origem Loop, which we call the Origem Arc, obviously has a nonthermal brightness temperature spectral index around $\beta \sim-2.7$ (flux density spectral index $\alpha=\beta+2=-0.7$ ). This region was once singled out by Krymkin \& Sidorchuk (1988) in their brightness temperature scans and designated as GR 0625+16. They suggested that GR $0625+16$ is a discrete SNR with an integrated radio spectral index of $\alpha=-0.48 \pm 0.05$. Although this spectral index is larger than the one we derived from our new data, both indicate the non-thermal nature of the Origem Arc.

The TT-plot method (Turtle et al. 1962) was used to verify the spectrum (see Fig. 3). The background point sources were subtracted first, as in Gao et al. (2011b). All images were then smoothed to a common angular resolution of 9.5. For the entire Origem Arc spanning $\ell=197^{\circ} .6$ to $\ell=192.1$ as indicated in Fig. 2, we obtained the spectral index of $\beta_{6-11}=-2.43 \pm 1.23$ from all the data pixels of $\lambda 6 \mathrm{~cm}$ and $\lambda 11 \mathrm{~cm}$, and $\beta_{6-21}=$ $-2.70 \pm 0.28$ for $\lambda 6 \mathrm{~cm}$ and $\lambda 21 \mathrm{~cm}$. Although the temperature measurements for each pixel are not independent, the TTplots give the correct brightness temperature spectral indices and the uncertainty estimates, as we tested by using the independent pixels. The TT-plot of the brighter part (high signal-tonoise ratio) of the arc $\left(\ell \geq 195^{\circ} .0\right)$ gives consistent results as $\beta_{6-11}=-2.45 \pm 1.06$, and $\beta_{6-21}=-2.65 \pm 0.29$. All these spectral values agree well with the spectral index map shown in Fig. 1d.

The other parts of the Origem Loop have different properties. The well-known H II region, BFS 52 (Blitz et al. 1982), has the central coordinates of $\ell=191.90, b=0.85$, and the quasar J061357.6+130645 (Aslan et al. 2010) is located at $\ell=197^{\circ} .00, b=-2.15$. Both have a flat spectrum $(\beta \sim-2.1)$. A circular region located at $\ell=195^{\circ} .60, b=-2.95$ with a diameter of $1^{\circ}$ was found to be very interesting. It has a brightness temperature spectral index of about $\beta \sim-2.5$ according to Fig. 1d. The TT-plot of this region gives a consistent result of $\beta_{6-21}=-2.33 \pm 0.23$, but this also implies a possibility of being a flat-spectrum thermal emission. We assign its name G195.60-2.95 and mark it using a circle with a central " $N$ " in 

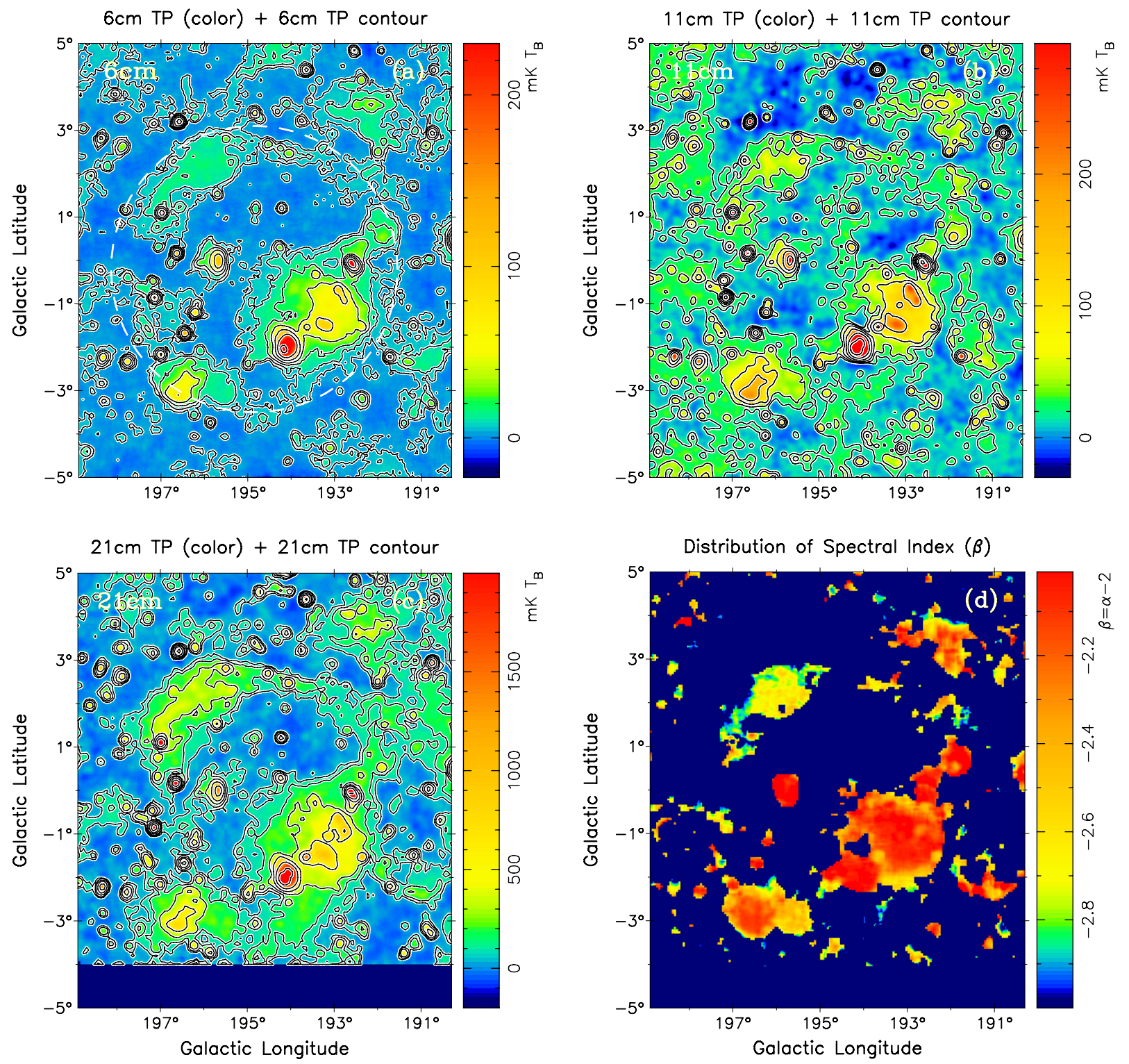

Fig. 1. From top left a), top right b) to bottom left panel $\mathbf{c}): \lambda 6 \mathrm{~cm}, \lambda 11 \mathrm{~cm}$, and the $\lambda 21 \mathrm{~cm}$ total intensity images of the Origem Loop. The angular resolutions are 9'.5, 9'.5, and 9'.4, respectively. The contours run from $2^{n} \times 3.6(3 \sigma) \mathrm{mK} T_{\mathrm{B}},(n=0,1,2 \ldots)$ for the $\lambda 6 \mathrm{~cm}$ image, $2^{n} \times 18.0(3 \sigma) \mathrm{mK} T_{\mathrm{B}},(n=0,1,2 \ldots)$ for the $\lambda 11 \mathrm{~cm}$ image, and $2^{n} \times 66.0(3 \sigma) \mathrm{mK} T_{\mathrm{B}},(n=0,1,2 \ldots)$ for the $\lambda 21 \mathrm{~cm}$ image. The white circle in the top left panel a) indicates the boundary of the Origem Loop. Bottom right panel d): spectral index distribution for the Origem Loop area derived from the Urumqi $\lambda 6 \mathrm{~cm}$, Effelsberg $\lambda 11 \mathrm{~cm}$, and $\lambda 21 \mathrm{~cm}$ images at the same angular resolution of 9.5.

Figs. 2 and 4. It has strong $\mathrm{H} \alpha$ emission and ring-shaped dust emission (see Figs. 4 and 7). The high ratio between the $60 \mu \mathrm{m}$ infrared and the $\lambda 6 \mathrm{~cm}$ continuum emission $(\sim 1400)$ indicates it as a probable thermal H II region. G192.8-1.1 has a flat thermal spectrum and is not an SNR, as discussed in Gao et al. (2011a).

The TT-plot can be used to reveal different emitting components with different spectral indices (e.g. Xiao et al. 2008). The TT-plot of the southern half of the Origem Loop shows that thermal emission is overwhelmingly dominant. Unlike in the northern arc, no evidence was found for any detectable unambiguous non-thermal emission component in the south (see Fig. 5).

\subsection{Polarization}

Observations of polarized emission at $\lambda 6 \mathrm{~cm}, \lambda 1.3 \mathrm{~cm}$, and DRAO $\lambda 21 \mathrm{~cm}$ were available for the Origem Loop region. However, only the $\lambda 6 \mathrm{~cm}$ data show the weak polarized emission associated with the Origem Arc (see the left panel of Fig. 4), in addition to the diffuse polarized background emission in the lower part of the map. At $\lambda 6 \mathrm{~cm}$, the polarized emission is clearly detected within the arc even at the western end where the total intensity becomes very weak. The polarization fraction is about $40 \%$ on average. The polarization B-field vectors $\left(\boldsymbol{E}+90^{\circ}\right)$ are found to follow the arc, indicating the presence of tangential magnetic fields. We also noticed that the depolarization zones seen at $\lambda 6 \mathrm{~cm}$ are correlated with the enhanced $\mathrm{H} \alpha$ emission (see the right panel of Fig. 4), e.g. the area around $\ell \sim 192^{\circ} .0, b \sim 3^{\circ} .0$, probably due to the Faraday rotation caused by the magnetic fields and the thermal electrons. A shuttle-shaped depolarization zone is found to cross the Origem Arc from northwest to southeast, where bright $\mathrm{H} \alpha$ filaments have good positional correspondences and morphological similarities.

In the southern part of the Origem Loop region, a few large polarization patches were detected within and outside the loop. 


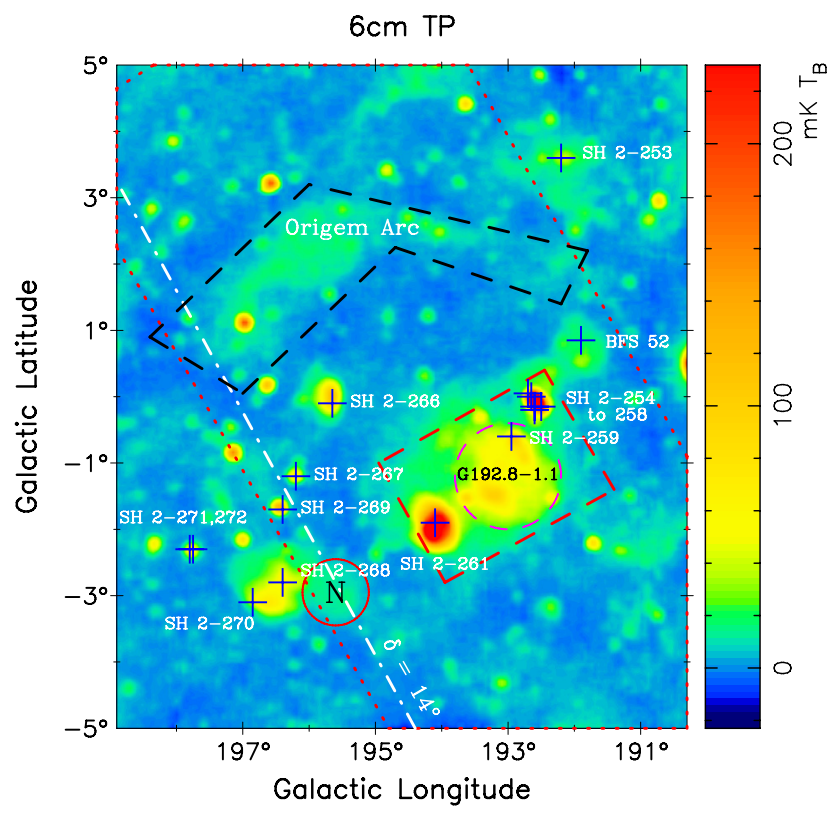

Fig. 2. $\lambda 6 \mathrm{~cm}$ total intensity image with the prominent $\mathrm{H}$ II regions marked with "+" and labelled with the names. The disproved SNR, G192.8-1.1, was also marked with a circle of the pink dashed line. The outer red dotted line delineates the common area of the observations by Krymkin \& Sidorchuk (1988) and our image, while the red dashed line indicates the field shown in Caswell (1985). The white dashed-dot line shows the declination of $\delta=14^{\circ}$ (Epoch 1950). The region on the left side of this line was not included in the $178 \mathrm{MHz}$ map used by Berkhuijsen (1974). The area outlined by the black dashed line, containing the Origem Arc, was used for the TT-plot in Sect. 3.1. A probable new H II region G195.60-2.95 is marked using a circle with the letter "N" inside.

However, none of them seems to be related either to the Origem Loop or G192.8-1.1 (Gao et al. 2011a). No arc-shaped structure in polarization can be found. In the area of $\ell=194^{\circ} 10, b=$ -1.85 , the H II region SH 2-261 acts as a Faraday screen.

At $\lambda 1.3 \mathrm{~cm}$, no polarized emission is visible in the entire area of the Origem Loop. Using the average brightness temperature of the polarized emission in the arc at $\lambda 6 \mathrm{~cm}, 5.0 \mathrm{mK} T_{\mathrm{b}}$, and the spectral index of $\beta=-2.70$, we estimated the brightness temperature of the polarized emission of the arc at $\lambda 1.3 \mathrm{~cm}$ to be about $0.07 \mathrm{mK} T_{\mathrm{b}}$. It is about the same level as the noise in the $K$-band data, which could account for the non-detection of polarization.

At $\lambda 21 \mathrm{~cm}$, no correlated polarized emission was detected in the Origem Arc from the DRAO data, either. The beam size of the DRAO data is $36^{\prime}$. Beam and depth depolarization could diminish any polarized emission. The non-detection might also imply a very near polarization horizon at $\lambda 21 \mathrm{~cm}$ in this direction.

\subsection{Distances of the Origem Arc and H II regions}

The observed tangential magnetic fields within the Origem Arc and the non-thermal spectrum are key evidence for identifying it as an SNR. To verify that this SNR and the H II regions located in the southern part of the Origem Loop are physically related, we need to know their distances first.

Despite the large uncertainty, the empirical relation between surface brightness and diameter $(\Sigma-\mathrm{D})$ of SNRs provides distance estimates of shell-type SNRs in case no related HI or
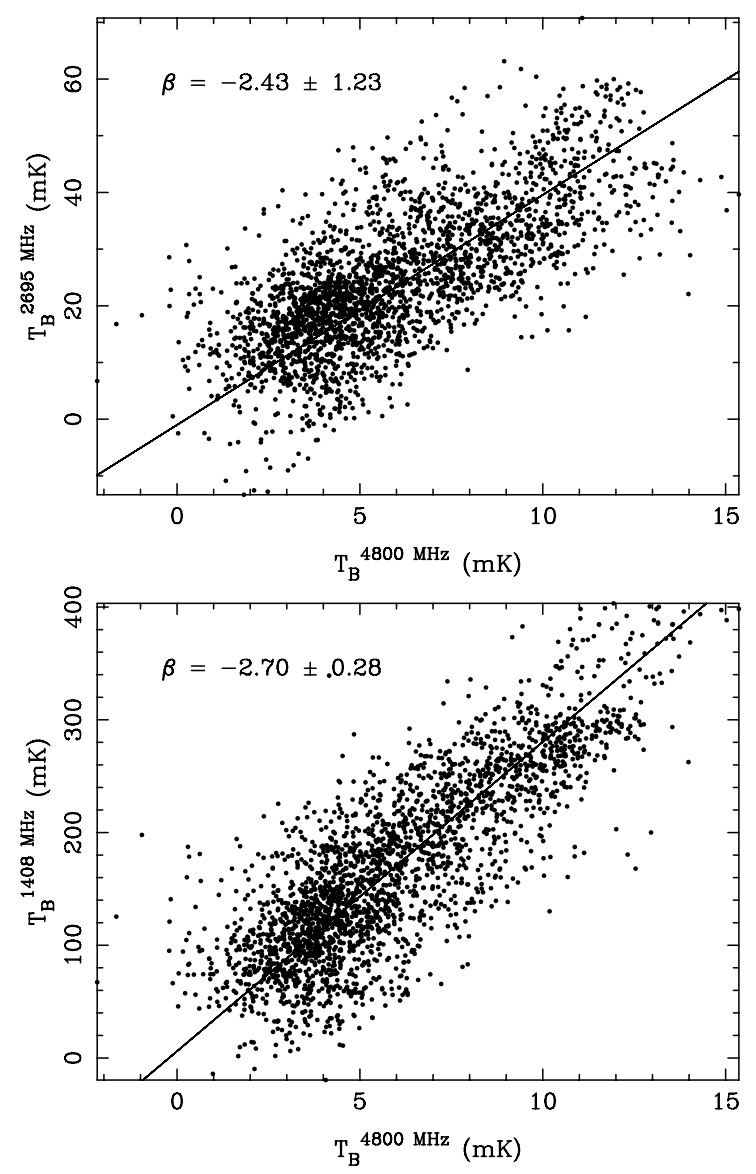

Fig. 3. TT-plot for all the pixels in the Origem Arc region (outlined by the black dashed line in Fig. 2) between $\lambda 6 \mathrm{~cm}$ and $\lambda 11 \mathrm{~cm}$ (upper panel) and between $\lambda 6 \mathrm{~cm}$ and $\lambda 21 \mathrm{~cm}$ (bottom panel).

molecular clouds (MC) are associated with the SNR. For the entire Origem Arc, a sector with an opening angle of $128^{\circ}$, the flux density at $\lambda 6 \mathrm{~cm}$ is measured to be $S_{6 \mathrm{~cm}}=8.5 \pm 0.9 \mathrm{Jy}$ after subtracting the background sources. We extrapolate $S_{6 \mathrm{~cm}}$ to the flux density at $1 \mathrm{GHz}$ with the spectral index of $\alpha=-0.7$. Using the arc radius of 3.3 measured on the map, we obtained a radio surface brightness of the Origem Arc of $\Sigma_{1 \mathrm{GHz}}=(8.6 \pm 0.9) \times$ $10^{-23} \mathrm{Wm}^{-2} \mathrm{~Hz}^{-1} \mathrm{sr}^{-1}$. According to the $\Sigma$-D relation found by Case \& Bhattacharya (1998), the diameter of the Origem Loop is estimated to be about $195 \mathrm{pc}$ and the distance to be about $1.7 \mathrm{kpc}$. As emphasized by Case \& Bhattacharya (1998), the deviation of an individual estimate derived from their work can be as large as $40 \%$, which puts the Origem Arc within a distance range between 1.0 and $2.4 \mathrm{kpc}$. Asvarov (2006) built a model that describes the evolution of the surface brightness and the diameter of shell-type SNRs with time. From his $\Sigma$-D relation (see his Fig. 6), we estimated that the Origem Arc has a diameter between 100 and $300 \mathrm{pc}$, corresponding to a distance range of 0.9 to $2.6 \mathrm{kpc}$. We therefore conclude that the Origem Arc is likely to have a distance of $1.7 \pm 0.8 \mathrm{kpc}$.

The average kinematic distance of several H II regions located in the southern part of the Origem Loop was found to be $1.2 \pm 0.7 \mathrm{kpc}$ (Berkhuijsen 1974). However, as noted by Caswell (1985), the kinematic distances have large uncertainties. More accurate distance determinations towards these $\mathrm{H}$ II regions have been obtained through the photometric and trigonometric parallax measurements as we listed in Table 2, except for SH 2-270. The lower half of the Origem Loop consists of the H II regions 

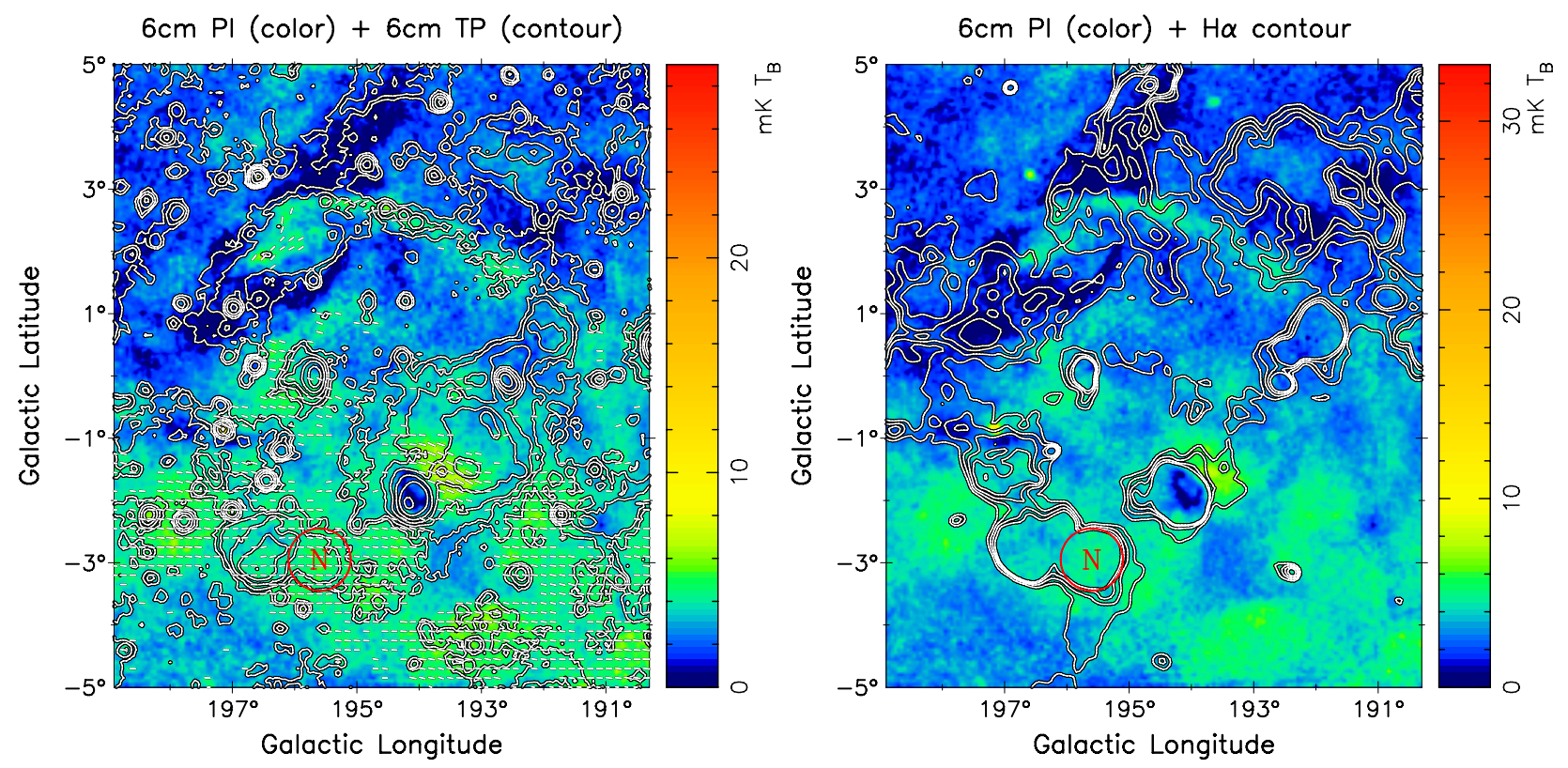

Fig. 4. left panel: $\lambda 6 \mathrm{~cm}$ polarization image (9'.5 resolution) of the Origem Loop region superimposed with total intensity contours and polarization vector $\boldsymbol{B}=\boldsymbol{E}+90^{\circ}$. right panel: $\lambda 6 \mathrm{~cm}$ polarization image overlaid with the $\mathrm{H} \alpha$ intensity (6' resolution) contours. Red circles with "N" inside in both images indicate a probable new $\mathrm{H}$ II region, see detail in the text.

BFS 52, SH 2-254 to 258, SH 2-261, SH 2-268, and the H II region SH 2-266, which have similar distances to the Origem Arc, and the other distant $\mathrm{H}$ II regions SH 2-253, SH 2-267, SH 2-269, SH 2-271, and SH 2-272. We could not get the distances of the object G192.8-1.1 and the newly identified object G195.60-2.95.

\subsection{Signatures at other wavelengths}

Berkhuijsen (1974) searched for possible H I structures associated with the Origem Loop, however, no clue was found. Denoyer et al. (1977) proposed that an Hi jet, which appears at $\ell \sim 197^{\circ}, b \sim 2^{\circ}$ may be related to the Origem Arc, although the jet is extended beyond its boundary. This jet is a part of the prominent H I structure, the anticentre shell (ACS), which was discovered by Heiles (1984). We checked the new H I data from the Leiden/Argentina/Bonn H I survey (Hartmann \& Burton 1997; Kalberla et al. 2005) and the GALFA H I DR1 data $^{4}$ for a much larger area $\left(20^{\circ} \times 20^{\circ}\right)$. We found that the ACS is prominent in the negative velocity map and disappears in the positive velocity map. This clearly differs from the positive $\mathrm{CO}$ radial velecity associated with the $\mathrm{H}$ II regions discussed above. Moreover, the ACS has a much larger size $\left(\sim 30^{\circ}\right.$ in diameter $)$ than the Origem Loop. For the velocity 0.0 to $32.5 \mathrm{~km} \mathrm{~s}^{-1}$, no associated H I structure is found around the Origem Loop.

Berkhuijsen (1974) investigated the relation between the Origem Loop and the H II regions. Based on the age of the loop and the evolution timecale from protostars to the H II regions, it was hard to tell whether the Origem Loop triggered the star formation that leads to the $\mathrm{H}$ II regions in the south. Using the physical size determined in this work, adopting Eq. (3) in Berkhuijsen (1974), the Origem Arc is about 1 to 3 Myr old. Chavarría et al. (2008) estimated the ages of the H II regions SH 2-254 to 258 from an expansion model of a Strömgren sphere, ranging between 0.1 Myr to 5.0 Myr. Bieging et al. (2009) have proposed a sequential star formation scenario from interaction between

\footnotetext{
4 https://purcell.ssl. berkeley.edu/index.php
}

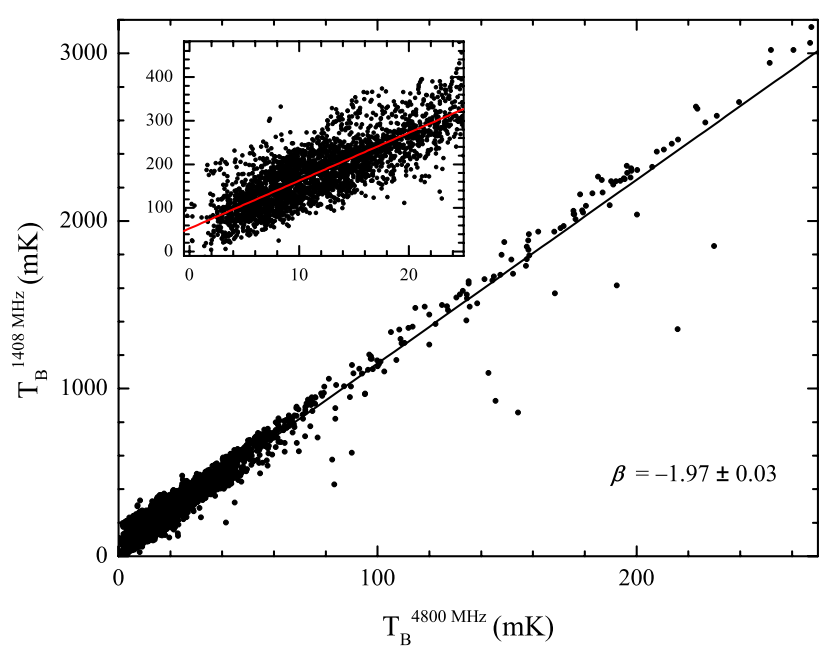

Fig. 5. TT-plot for the southern part of the Origem Loop between $\lambda 6 \mathrm{~cm}$ and $\lambda 21 \mathrm{~cm}$. The inner small image is the zoom-in picture for the value $T_{6 \mathrm{~cm}} \leqslant 25 \mathrm{mK} T_{\mathrm{b}}$. The red line in the small image represents the same spectral index as in the large image.

the $\mathrm{H}$ II regions and molecular clouds, which indicates that at least the younger $\mathrm{H}$ II regions $\mathrm{SH} 2-255$ to 257 were triggered by SH 2-254.

The interaction between the SNR shock and the ambient molecular clouds should broaden the linewidth. For example, IC 443, a famous SNR-MC interaction case, clearly shows a high $\mathrm{CO} J=3-2 / \mathrm{CO} J=2-1$ ratio and the broadening of $\mathrm{CO}$ emission lines (Zhang et al. 2010; Xu et al. 2011). Broadened CO $J=1-0$ lines were also detected in another SNR-MC interaction case (Byun et al. 2006). Public CO $J=3-2$ and CO $J=$ $2-1$ data covering the Origem Loop region are not available. We checked the CO $J=1-0$ data of the Origem Loop region from the CO survey ${ }^{5}$ (Dame et al. 2001). The radial velocities of the

5 http://www.cfa.harvard.edu/rtdc/CO/ 
Table 2. Distance of the known H II regions.

\begin{tabular}{|c|c|c|c|c|c|c|c|c|}
\hline $\begin{array}{c}\text { Name } \\
\text { (1) }\end{array}$ & $\begin{array}{l}\ell \\
\left({ }^{\circ}\right) \\
(2)\end{array}$ & $\begin{array}{l}b \\
\left({ }^{\circ}\right) \\
(3)\end{array}$ & $\begin{array}{c}\text { CO velocity } \\
\left(\mathrm{km} \mathrm{s}^{-1}\right) \\
(4)\end{array}$ & $\begin{array}{c}\text { Distance (B74) } \\
(\mathrm{kpc}) \\
(5)\end{array}$ & $\begin{array}{c}\text { Distance (C85) } \\
(\mathrm{kpc}) \\
(6)\end{array}$ & $\begin{array}{l}\text { Recent distance } \\
(\mathrm{kpc}) \\
(7)\end{array}$ & $\begin{array}{c}\text { Method } \\
\text { see notes } \\
\text { (8) }\end{array}$ & References \\
\hline BFS 52 & $191^{\circ} .90$ & $+0^{\circ} 85$ & $7.3 \pm 0.5$ & $\cdots$ & $\cdots$ & $2.10_{-0.026}^{+0.027} / 1.59_{-0.06}^{+0.07}$ & $\mathrm{p}^{*}$ & $1 / 2$ \\
\hline SH 2-253 & $192^{\circ} .23$ & +3.59 & $14.4 \pm 0.5$ & .. & $\ldots$ & $5.1 \pm 1.5$ & s & 3 \\
\hline SH 2-254 & $192^{\circ} .49$ & -0.15 & $7.5 \pm 0.7$ & $1.12 \pm 0.92$ & 2.5 & $1.59_{-0.06}^{+0.07}$ & $\mathrm{p}^{\dagger}$ & 2 \\
\hline SH 2-255 & 192.64 & -0.01 & $7.5 \pm 0.7$ & $0.88 \pm 0.80$ & 2.5 & $1.59_{-0.06}^{+0.07}$ & $\mathrm{p}$ & 2 \\
\hline SH 2-256 & $192^{\circ} .62$ & -0.13 & $7.5 \pm 0.7$ & $\ldots$ & 2.5 & $1.59_{-0.06}^{+0.07}$ & $\mathrm{p}^{\dagger}$ & 2 \\
\hline SH 2-257 & $192^{\circ} .61$ & -0.07 & $7.5 \pm 0.7$ & $1.34 \pm 0.95$ & $\ldots$ & $1.59_{-0.06}^{+0.00}$ & $\mathrm{p}^{\dagger}$ & 2 \\
\hline SH 2-258 & $192^{\circ} .73$ & $+0^{\circ} 05$ & $7.5 \pm 0.7$ & $\ldots$ & 2.5 & $1.59_{-0.06}^{+0.07}$ & $\mathrm{p}^{\dagger}$ & 2 \\
\hline SH 2-259 & $192^{\circ} .94$ & -0.58 & $22.8 \pm 0.5$ & .. & 8.3 & $8.9 \pm 2.7$ & $\mathrm{~s}$ & 3 \\
\hline SH 2-261 & $194^{\circ} .10$ & -1.90 & $\ldots$ & $0.90 \pm 0.80$ & $2.3 / 2.1$ & $1.6 \pm 0.5$ & $\mathrm{~s}$ & 3 \\
\hline SH 2-266 & $195^{\circ} 66$ & -0.08 & $31.2 \pm 1.1$ & $\cdots$ & $\cdots$ & $2.0 \pm 0.6$ & $\mathrm{~s}$ & 3 \\
\hline SH 2-267 & $196^{\circ} .20$ & -1.20 & $\ldots$ & $\ldots$ & $\ldots$ & $\sim 4.2$ & $\mathrm{~s}$ & 4 \\
\hline SH 2-268 & $196^{\circ} .40$ & -2.80 & $4.8 \pm 0.5$ & $\ldots$ & $\ldots$ & $1.3 \pm 0.4$ & $\mathrm{~s}$ & 3 \\
\hline SH 2-269 & $196^{\circ} .40$ & -1.70 & $17.5 \pm 0.7$ & $1.88 \pm 0.80$ & $\ldots$ & $5.28_{-0.22}^{+0.24}$ & $\mathrm{p}$ & 5 \\
\hline SH 2-270 & $196^{\circ} 84$ & -3.11 & $25.6 \pm 0.4$ & $\ldots$ & $\ldots$ & $6.8 \pm 2.3$ & $\mathrm{k}$ & 3 \\
\hline SH 2-271 & 197.77 & $-2^{\circ} .31$ & $20.5 \pm 0.5$ & $\ldots$ & $\ldots$ & $5.1 \pm 1.1$ & $\mathrm{~s}$ & 3 \\
\hline SH 2-272 & 197.81 & -2.28 & $20.5 \pm 0.5$ & $\ldots$ & $\ldots$ & $5.1 \pm 1.1$ & $\mathrm{~s}$ & 3 \\
\hline
\end{tabular}

Notes. The source names are listed in Col. (1) and the central coordinates are in Cols. (2) and (3). The radial velocities of the peak CO emission associated with the H II regions are given in Col. (4). Columns (5) and (6) are the distances in Berkhuijsen (1974, B74) and Caswell (1985, C85). The distances taken from the recent literature are listed in Col. (7) with the distance measurement method given in Col. (8), and the references are given in Col. (9).

p: parallax; $\mathrm{p}^{*}$ : associated with SH 2-252 or SH 2-254; p†: SH 2-254 to 258 are regarded to have the same distance; s: stellar distance; k: kinematic distance.

References. (1) Reid et al. (2009), (2) Rygl et al. (2010), (3) Russeil (2003), (4) Lahulla (1987), (5) Honma et al. (2007).
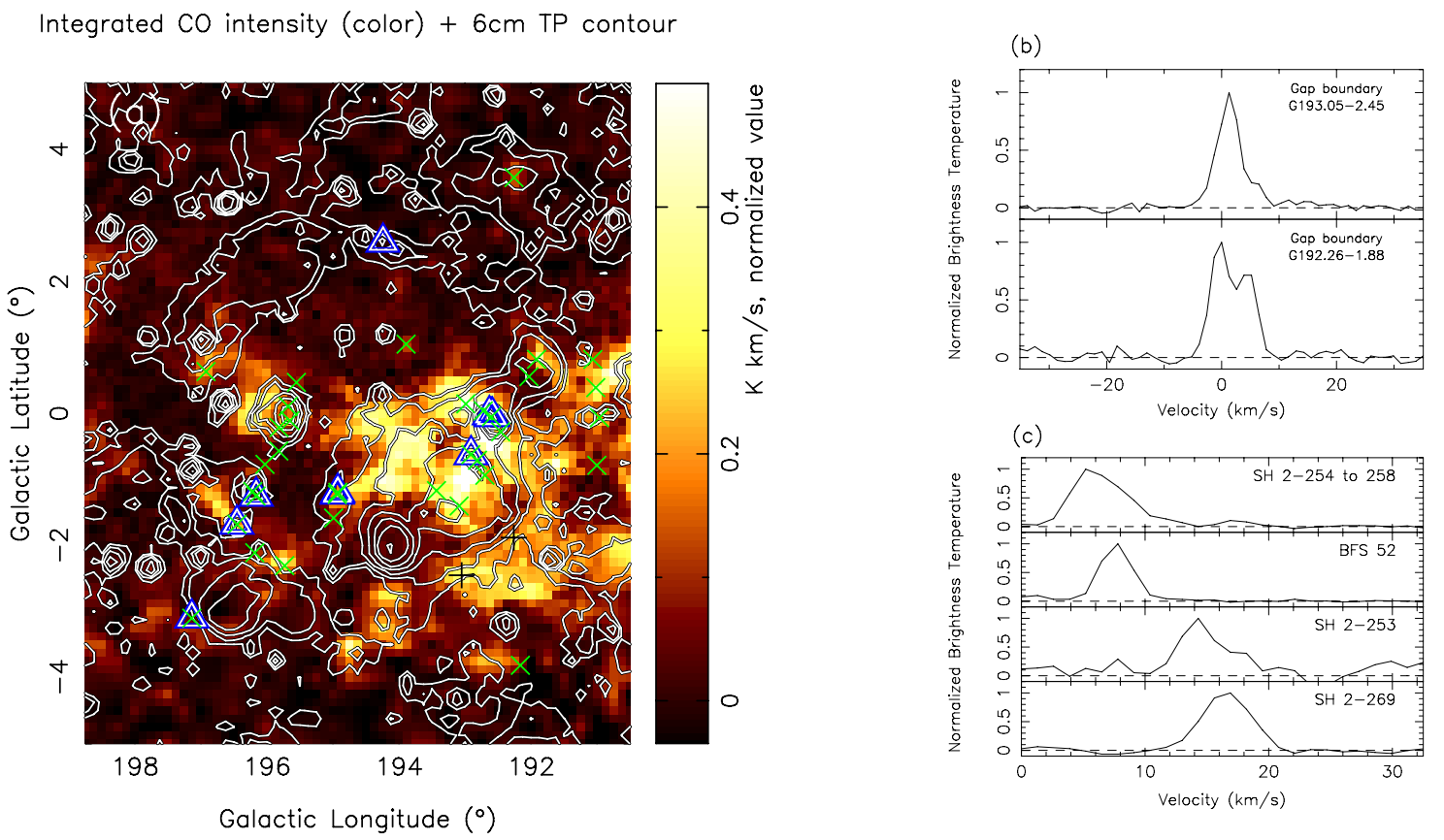

Fig. 6. Left panel: $\mathrm{CO}$ intensity map integrated from 0.0 to $32.5 \mathrm{~km} \mathrm{~s}^{-1}$, overlaid by the $\lambda 6 \mathrm{~cm}$ total intensity contours as shown in Fig. 1 . Each pixel value was normalized by dividing by the maximum integrated intensity in the image. The green crosses represent the proto-stellar candidates selected from the IRAS point source catalogue by the criteria introduced by Junkes et al. (1992), while the blue-white triangles are the massive young stellar objects found in the Red MSX survey. Top right panel: normalized CO emission profile for the two areas marked with the black "plus" in the left panel. Bottom right panel: CO spectra for the H II regions SH 2-254 to 258, BFS 52, SH 2-253, and SH 2-269. The brightness temperature in each region was also normalized. 


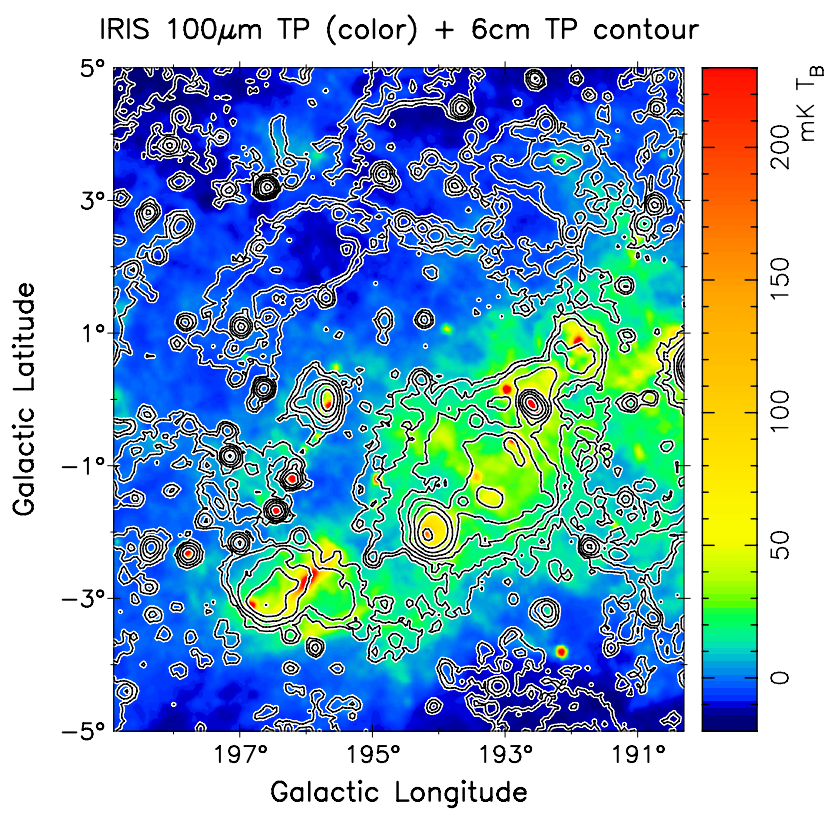

Fig. 7. $100 \mu \mathrm{m}$ dust map ( $2^{\prime}$ resolution) overlaid with the contours of the $\lambda 6 \mathrm{~cm}$ total intensity. The contours are the same as in Fig. 1.

$\mathrm{CO}$ emission peaks associated with these $\mathrm{H}$ II regions were previously measured and given by Blitz et al. (1982), as we listed in our Table 2. Therefore, we integrated the $\mathrm{CO}$ emission in the velocity range from $0.0 \mathrm{~km} \mathrm{~s}^{-1}$ to $32.5 \mathrm{~km} \mathrm{~s}^{-1}$ and show the result in the left-hand panel of Fig. 6. We find that the most intense CO emission comes from the area of G192.8-1.1. It has a roughly similar morphology to the $\lambda 6 \mathrm{~cm}$ continuum emission as illustrated by the contour lines in Fig. 6. An obvious gap of CO emission is seen at the edge of the continuum emission in the southwest of G192.8-1.1. We checked the velocity-intensity relation at two positions indicated by "plus" in the left-hand panel of Fig. 6 and also the velocity-intensity plots for the H II region SH 2-254 to SH 2-258, BFS 52 for the possible interaction group and the H II regions SH 2-253, SH 2-269 for the non-interaction group. As shown in the right-hand panel of Fig. 6, all of the radial velocities corresponding to the peak $\mathrm{CO}$ emission for the H II regions are consistent with those found by Blitz et al. (1982). We checked the linewidths for several hundred non-interacting H II regions (Anderson et al. 2009; Russeil \& Castets 2004) and found the average values are in the range of $3 \sim 6 \mathrm{~km} \mathrm{~s}^{-1}$. SH 2-254 to 258, BFS 52, SH 2-253, and SH 2-269 have similar linewidths, and no significant rise in the line wings can be seen. Therefore there are no hints for the interaction between SNR and clouds for $\mathrm{H}$ II region formation.

We searched for massive young stellar objects (YSOs) in the Origem Loop region from the Red MSX Source Survey database $^{6}$ and for the protostellar candidates in the IRAS point source catalogue. They are marked in Fig. 6. Several of them are coincident and most of them are located in the regions where $\mathrm{CO}$ emission is prominent. The YSOs in the Origem Loop region do not show over density on the northern Origem Arc or on the shell-like structure where the continuum-CO boundary exists.

Infrared (dust) image was also checked by Berkhuijsen (1974), nothing coincided with the Origem Arc (see her Fig. 3). From the IRIS $100 \mu \mathrm{m}$ dust image (Miville-Deschênes \& Lagache 2005) of the Origem Loop region shown in Fig. 7, we found that the dust emission is well correlated with the

\footnotetext{
6 http://www.ast.leeds.ac.uk/RMS/
}

$\mathrm{H}$ II regions in the south. The two interacting $\mathrm{H}$ II regions SH 2-255 and SH 2-257 triggered star formation in between them (Ojha et al. 2011). A large number of YSOs were identified on their boundary. The object G195.60-2.95 has an incomplete ring structure, and the infrared emission gets enhanced between it and the known $\mathrm{H}$ II regions $\mathrm{SH} 2-268$, which is about $1.3 \mathrm{kpc}$ away. However, we do not get any YSOs on the intensive infrared emission zone. This might be due to limitation of the infrared data sets we use. The apparent sizes of the infrared bubbles in the inner Galaxy are generally smaller than the one around G195.60-2.95 (Simpson et al. 2012), which may imply a small distance to G195.60-2.95. An infrared loop was also found around the SNR (Koo et al. 2008). However, the polarization measurement at $\lambda 6 \mathrm{~cm}$ and the infrared/radio ratio of G195.60-2.95 strongly suggest that it is an H II region.

We checked the $0.1-0.4 \mathrm{keV}$ and $0.4-2.4 \mathrm{keV}$ ROSAT X-ray images $^{7}$, and no associated structure with the Origem Loop was found.

\section{Summary}

We used multi-frequency survey data to revisit the Origem Loop in the anticentre of the Galaxy. The Origem Arc is a polarized non-thermal emission structure with a spectral index of $\beta=-2.70$, indicating that it is a shell-type SNR. We estimated its distance to be $1.7 \pm 0.8 \mathrm{kpc}$.

Using the new radio data, we discussed the possibilities of a physical association between the SNR and the H II regions located in the south of the Origem Loop. The TT-plots for different emission components, the width of $\mathrm{CO}$ lines, and age estimates did not show any evidence of a non-thermal southern arc or the interaction between the SNR and the HII regions in the south. Associated infrared emission is seen to be closely related to the $\mathrm{H}$ II regions in the southern part of the loop. No H I or X-ray emission correlated with the Origem Loop was found.

Acknowledgements. We would like to thank the referee, Dr. Elly Berkhuijsen, for constructive and helpful comments. The Sino-German $\lambda 6 \mathrm{~cm}$ polarization survey was carried out with a receiver system constructed by Mr. Otmar Lochner at MPIfR mounted at the Nanshan 25-m telescope of the Urumqi Observatory of NAOC. The MPG and the NAOC/CAS supported the construction of the receiving system by special funds. We thank Mr. Maozheng Chen and Mr. Jun Ma for qualified maintenance of the receiving system for many years. The authors are supported by the National Natural Science foundation of China (10833003) and the Partner group of the MPIfR at NAOC in the framework of the exchange program between MPG and CAS for many bilateral visits. X.Y.G. is additionally supported by the Young Researcher Grant of National Astronomical Observatories, Chinese Academy of Sciences. This paper made use of information from the Red MSX Source survey database at www.ast. leeds.ac.uk/RMS, which was constructed with support from the Science and Technology Facilities Council of the UK. This paper also utilizes data from Galactic ALFA HI (GALFA HI) survey data set obtained with the Arecibo $L$ band Feed Array (ALFA) on the Arecibo $305 \mathrm{~m}$ telescope. Arecibo Observatory is part of the National Astronomy and Ionosphere Center, which is operated by Cornell University under Cooperative Agreement with the US National Science Foundation. The GALFA HI surveys are funded by the NSF through grants to Columbia University, the University of Wisconsin, and the University of California.

\section{References}

Anderson, L. D., Bania, T. M., Jackson, J. M., et al. 2009, ApJS, 181, 255

Aslan, Z., Gumerov, R., Jin, W., et al. 2010, A\&A, 510, A10

Asvarov, A. I. 2006, A\&A, 459, 519

Berkhuijsen, E. M. 1974, A\&A, 35, 429

Berkhuijsen, E. M., Haslam, C. G. T., \& Salter, C. J. 1971, A\&A, 14, 252

\footnotetext{
http://www . xray.mpe.mpg.de/cgi_bin/rosat/ rosat_survey
} 
Bieging, J. H., Peters, W. L., Vila Vilaro, B., Schlottman, K., \& Kulesa, C. 2009, AJ, 138,975

Blitz, L., Fich, M., \& Stark, A. A. 1982, ApJS, 49, 183

Byun, D.-Y., Koo, B.-C., Tatematsu, K., \& Sunada, K. 2006, ApJ, 637, 283

Case, G. L., \& Bhattacharya, D. 1998, ApJ, 504, 761

Caswell, J. L. 1985, AJ, 90, 1076

Caswell, J. L., \& Crowther, J. H. 1969, MNRAS, 145, 181

Chavarría, L. A., Allen, L. E., Hora, J. L., Brunt, C. M., \& Fazio, G. G. 2008, ApJ, 682, 445

Dame, T. M., Hartmann, D., \& Thaddeus, P. 2001, ApJ, 547, 792

Denoyer, L. K., Button, L., Chaffin, D., \& Nieznanski, J. 1977, ApJ, 213, 379

Fürst, E., Reich, W., Reich, P., \& Reif, K. 1990, A\&AS, 85, 691

Gao, X. Y., Reich, W., Han, J. L., et al. 2010, A\&A, 515, A64

Gao, X. Y., Han, J. L., Reich, W., et al. 2011a, A\&A, 529, A159

Gao, X. Y., Sun, X. H., Han, J. L., et al. 2011b, A\&A, 532, A144

Gardner, F. F., \& Milne, D. K. 1965, AJ, 70, 754

Green, D. A. 2009, Bull. Astron. Soc. India, 37, 45

Hanbury Brown, R., Davies, R. D., \& Hazard, C. 1960, The Observatory, 80, 191

Hartmann, D., \& Burton, W. B. 1997, Atlas of Galactic Neutral Hydrogen, eds.

D. Hartmann, \& W. B. Burton

Haslam, C. G. T., Kahn, F. D., \& Meaburn, J. 1971, A\&A, 12, 388

Heiles, C. 1984, ApJS, 55, 585

Honma, M., Bushimata, T., Choi, Y. K., et al. 2007, PASJ, 59, 889

Jarosik, N., Bennett, C. L., Dunkley, J., et al. 2011, ApJS, 192, 14

Junkes, N., Fuerst, E., \& Reich, W. 1992, A\&A, 261, 289

Kalberla, P. M. W., Burton, W. B., Hartmann, D., et al. 2005, A\&A, 440, 775

Koo, B.-C., McKee, C. F., Lee, J.-J., et al. 2008, ApJ, 673, L147
Krymkin, V. V., \& Sidorchuk, M. A. 1988, A\&A, 200, 185

Lahulla, J. F. 1987, AJ, 94, 1062

Large, M. I., Quigley, M. J. S., \& Haslam, C. G. T. 1962, MNRAS, 124, 405

Large, M. I., Quigley, M. F. S., \& Haslam, C. G. T. 1966, MNRAS, 131, 335

Miville-Deschênes, M., \& Lagache, G. 2005, ApJS, 157, 302

Ojha, D. K., Samal, M. R., Pandey, A. K., et al. 2011, ApJ, 738, 156

Quigley, M. J. S., \& Haslam, C. G. T. 1965, Nature, 208, 741

Reich, P., Reich, W., \& Fürst, E. 1997, A\&AS, 126, 413

Reid, M. J., Menten, K. M., Brunthaler, A., et al. 2009, ApJ, 693, 397

Russeil, D. 2003, A\&A, 397, 133

Russeil, D., \& Castets, A. 2004, A\&A, 417, 107

Rygl, K. L. J., Brunthaler, A., Reid, M. J., et al. 2010, A\&A, 511, A2

Simpson, R. J., Povich, M. S., Kendrew, S., et al. 2012, MNRAS, 424, 2442

Sofue, Y., \& Reich, W. 1979, A\&AS, 38, 251

Turtle, A. J., Pugh, J. F., Kenderdine, S., \& Pauliny-Toth, I. I. K. 1962, MNRAS, 124, 297

Uyanıker, B., Fürst, E., Reich, W., Reich, P., \& Wielebinski, R. 1998, A\&AS, 132,401

Uyanıker, B., Fürst, E., Reich, W., Reich, P., \& Wielebinski, R. 1999, A\&AS, 138,31

Walsh, D., \& Brown, R. H. 1955, Nature, 175, 808

Wolleben, M., Landecker, T. L., Reich, W., \& Wielebinski, R. 2006, A\&A, 448, 411

Xiao, L., Fürst, E., Reich, W., \& Han, J. L. 2008, A\&A, 482, 783

Xu, J.-L., Wang, J.-J., \& Miller, M. 2011, ApJ, 727, 81

Zhang, Z., Gao, Y., \& Wang, J. 2010, Sci. China G: Phys. Astron., 53, 1357 